\section{Todesursachen bei Patienten mit chronischer Lungenerkrankung}

Gayle AV et al. Changing causes of death for patients with chronic respiratory disease in England, 2005-2015. Thorax 2019; doi:10.1136/

thoraxjnl-2018-212514

Seit 1980 wird international eine um $30 \%$ gestiegene Mortalität aufgrund chronischer Lungenerkrankungen (CRD, Chronic Respiratory Disease) beobachtet. Das Vereinigte Königreich hat innerhalb der OECD (Organisation für wirtschaftliche $\mathrm{Zu}$ sammenarbeit und Entwicklung) die höchste respiratorische Mortalitätsrate. Britische Ärzte untersuchten die Todesursachen von CRD-Patienten und wie sich die Mortalität über 10 Jahren verändert hat.

Aus elektronischen Krankenakten des "Clinical Practice Research Datalink“ (CPRD) identifizierte die Arbeitsgruppe englische Patienten über 18 Jahre mit Asthma, Brochiektasien oder interstitieller Lungenerkrankung und COPD-Patienten über 35 Jahre. Der gewählte Zeitraum umfasste die Jahre 2005-2015. Die Nachbeobachtung begann spätestens zum Studienstart oder zum Zeitpunkt, an dem die behandelnde Praxis am CPRD teilnehmen konnte, oder wenn eine CRD erstmals diagnostiziert wurde. Verstorbene wurden als Asthmapatienten erachtet, wenn die letzte Asthmakodierung nicht länger als 3 Jahre vor dem Tod zurücklag. Die Mortalitätsraten bestimmten A. V. Gayle et al. für jede CRD und verglichen sie mit denen der englischen Allgemeinbevölkerung.

\section{Ergebnisse}

Insgesamt 558888 CRD-Patienten erfüllten die Einschlusskriterien, von denen 451830 Asthma, 137709 COPD, 19374 Bronchiektasien und 10745 interstitelle Lungenerkrankungen hatten. Im Beobachtungszeitraum verstarben $14 \%$ aus der Gesamtgruppe. Das durchschnittliche Alter zum Todeszeitpunkt stieg zwischen 2005 und 2015 von 77,4 auf 79,2 Jahre. Die altersstandardisierte Sterblichkeit der CRD-Patienten insgesamt betrug
1607 pro 100000 Personen und für Patienten mit

- Asthma 856,

- Bronchiektasie 1463,

- COPD 1503,

- interstitieller Lungenerkrankung 2609 pro 100000 Personen.

Damit war die altersstandardisierte Sterblichkeit um 54\% höher als in der Allgemeinbevölkerung. Zu den bedeutendsten Todesursachen bei CRD gehörten Erkrankungen der Atemwege (32\%), des Kreislaufsystems (26\%) und Neoplasien (24\%). COPD war mit 66\% die häufigste Ursache atemwegsbedingter Todesfälle bei allen CRD-Patienten, ausgenommen diejenigen mit interstitieller Lungenerkrankung. Innerhalb der CRD-Gesamtgruppe und bei einzelnen Lungenerkrankungen blieben die Sterblichkeitsraten aufgrund von Atemwegserkrankungen zwischen 2005 und 2015 unverändert. Dagegen reduzierte sich die Mortalität aufgrund von Kreislauferkrankungen von $30 \%$ (2005) auf $23 \%$ (2015).

\section{FAZIT}

Die Sterblichkeit ist in England bei Patienten mit chronischer Lungenerkrankung deutlich höher als in der Allgemeinbevölkerung. Innerhalb des 10-jährigen Beobachtungszeitraums verbesserte sich bei CRD-Patienten die atemwegsbedingte Sterblichkeit nicht.

Matthias Manych, Berlin 